\title{
Metodología del aprendizaje basado en problemas como una herramienta para el logro del proceso de enseñanza- aprendizaje.
}

Problem-based learning methodology as a tool for the achievement of the teaching-learning process.

\section{Metodología del aprendizaje basado en problemas.}

\section{Raquel Vera Velázquez ${ }^{(1)}$}

Kirenia Maldonado Zúñiga ${ }^{(2)}$

Carlos Castro Piguave ${ }^{(3)}$

Yoiler Batista Garcet ${ }^{(4)}$

${ }^{1}$ Universidad Estatal del Sur de Manabí. Jipijapa. Ecuador. email: raquelita2015vera@gmail.com ORCID: https://orcid.org/0000-0002-5071-7523

${ }^{2}$ Universidad Estatal del Sur de Manabí Jipijapa. Ecuador. email: kmzmaldonado@gmail.com ORCID: https://orcid.org/0000-0002-3764-5633

${ }^{3}$ Universidad Estatal del Sur de Manabí. Jipijapa. Ecuador. email: carlos.castro@unesum.edu.ec. Código ORCID: https://orcid.org/0000-0003-3180-2359

${ }^{4}$ Universidad Estatal del Sur de Manabí. Jipijapa. Ecuador. yoiler.batista@unesum.edu.ec. Código ORCID: https://orcid.org/0000-0002-7851-5763

Contacto: raquelita2015vera@gmail.com

\section{Resumen}

El Aprendizaje Basado en Problemas (ABP) es una de las metodologías educativas que han tenido buena aceptación en instituciones universitarias. Es un proceso activo de aprendizaje que funciona mediante la solución de problemas relacionados con la interacción de los estudiantes y su entorno profesional. La esencia del Aprendizaje Basado en Problemas consiste en identificar, describir, analizar y resolver tales problemas, lo cual se logra con ayuda del docente, desempeñando así otro papel tanto el proceso de enseñanza-aprendizaje como los estudiantes. Una forma en la que se ha incorporado recientemente esta metodología en el salón de clases de las carreras de ingeniería ha sido mediante trabajos teóricos que los estudiantes discuten y los resultados de los proyectos integradores 
de saberes que realizan en cada semestre. Por ejemplo, discuten los resultados de un artículo científico reciente en particular, del cual deben analizar los planteamientos presentados y que están relacionados con los problemas analizados y resueltos en las clases de matemática. El objetivo de esta investigación está dirigido a que el estudiante aprenda a resolver problemas mediante la búsqueda sistemática de información y el razonamiento científico, desarrollando temas contenidos en los programas de los cursos de matemáticas, utilizando para esto la Metodología del Aprendizaje Basado en Problemas (ABP) el logro principal es que los estudiantes presenten sus resultados en publicaciones de artículos, foros estudiantiles, en congresos nacionales, como producto de la aplicación del Aprendizaje Basado en Problemas en el desarrollo del proceso de enseñanza-aprendizaje.

Palabras clave: metodología, aprendizaje, proyectos, enseñanza, problemas.

\section{Summary}

Problem-Based Learning (PBL) is one of the educational methodologies that have been well accepted in university institutions. It is an active learning process that works by solving problems related to the interaction of students and their professional environment. The essence of Problem-Based Learning consists of identifying, describing, analyzing and solving such problems, which is achieved with the teacher's help, thus playing another role for both the teaching-learning process and the students. One way in which this methodology has recently been incorporated into the engineering careers classroom has been through theoretical work that students discuss and the results of the integrating knowledge projects that they carry out each semester. For example, they discuss the results of a recent scientific article in particular, of which they must analyze the proposals presented and that are related to the problems analyzed and solved in mathematics classes. The objective of this research is aimed at the student learning to solve problems through the systematic search for information and scientific reasoning, developing topics contained in the mathematics course syllabi, using the Problem-Based Learning Methodology (PBL). ) The main achievement is that students present their results in article publications, student forums, in national congresses, as a product of the application of Problem-Based Learning in the development of the teaching-learning process.

Keywords: methodology, learning, projects, teaching, problems. 


\section{Introducción}

Actualmente los estudiantes muestran desinterés y desmotivación hacia las matemáticas, siendo una de las principales causas la insuficiente aplicación de metodologías por parte del docente. La mayoría de los docentes de matemática aplican modelos de enseñanza en los cuales primero se aprenden contenidos y procesos para luego aplicarlos en problemas estructurados y en ejercicios de respuesta obligatoria con el objetivo de comprobar si se aprendió lo que se enseñó. Dichos métodos no permiten a los estudiantes adquirir los conocimientos necesarios a través del trabajo colaborativo e iniciativa propia de investigación, ni mucho menos aplicar en la solución de problemas de su vida diaria.

La asignatura de Matemática, ha sido considerada por los estudiantes como una de las materias más tediosas y difíciles, debido a que los docentes no han puesto énfasis en volverla accesible y motivadora. Por ello, han visto en la metodología Aprendizaje Basado en Problemas, un mecanismo pertinente para brindar a sus estudiantes una adecuada orientación sobre su uso, en la cual se busca la constante interacción entre el docente, estudiante y compañeros de clase. Además, permite entablar relaciones entre la realidad de los estudiantes con el contexto que los rodea, no solo a nivel educativo sino también a nivel personal Alzate, Montes, \& Escobar citados en Matamoros (2018).

El investigador Martínez (2014) define al Aprendizaje Basado en Problemas como un método de aprendizaje basado en el principio de emplear problemas como punto de partida para la adquisición e integración de los nuevos conocimientos. En esta metodología los protagonistas del aprendizaje son los propios estudiantes, que asumen la responsabilidad de ser parte activa en el proceso.

El Aprendizaje Basado en Problemas se sustenta con las teorías: constructivista y aprendizaje significativo según Mancheno (2013) expresa que el constructivismo es una postura psicológica y filosófica. Esta considera que los individuos forman o construyen gran parte de lo que aprenden. Menciona que esta teoría supone, que los individuos son participantes activos y que deben construir el conocimiento. Desde este punto de vista, los profesores no enseñan en el sentido tradicional, sino, acuden a materiales con los que el estudiante se compromete activamente mediante manipulación e interacción social y redescubren ellos mismos los principios básicos de estos materiales. 
El aprendizaje como un proceso activo: en el proceso de alojamiento y asimilación de la información, resultan vitales, la experiencia directa, las equivocaciones y la búsqueda de soluciones. La manera en la que se presenta la información es de suma importancia. Cuando la información es introducida como una forma de respuesta para solucionar un problema, funciona como una herramienta, no como un hecho arbitrario y solitario.

El aprendizaje es completo, auténtico y positivo: el significado es construido en la manera en que el individuo interactúa de forma significativa con el mundo que le rodea. Esto significa que se debe enfatizar en menor grado los ejercicios de habilidades solitarias, que intentan enseñar una lección. Los estudiantes que se encuentren en aulas diseñadas con este método llegan a aprender estas lecciones, pero les resulta más fácil el aprendizaje si al mismo tiempo se encuentran comprometidos con actividades significativas que ejemplifiquen lo que se desea aprender.

El constructivismo de Vygotsky, (1960) se enfoca sobre la base social del aprendizaje en las personas. El contexto social da a los estudiantes la oportunidad de llevar a cabo, de una manera más exitosa, habilidades más complejas que lo que pueden realizar por sí mismos. En los individuos, el componente social es muy importante, tener amigos y compartir con ellos.

Ausubel, (1986) aporta con su teoría al constructivismo señalando un modelo de enseñanza por exposición, promoviendo el aprendizaje significativo en lugar del aprendizaje memorístico. En el aprendizaje significativo, los nuevos conocimientos se incorporan en forma sustantiva en la estructura cognitiva del estudiante, cuando este relaciona los nuevos conocimientos con los anteriormente obtenidos Bonilla, (2015).

Desde una perspectiva constructivista, el papel del docente se orienta bajo la figura de mediador y propiciador de situaciones de aprendizaje, en las que el estudiante es considerado como participante activo del proceso, donde incluso se propicia que este dude de sus propias ideas y sienta la necesidad de buscar nuevas explicaciones, distintos caminos que vuelvan a satisfacer esos esquemas mentales, los cuales han sido configurados por la interacción con su medio natural y social Rojas, Urdaneta \& Guevara, (2014). 
Existen diversos contextos en los que se ha aplicado la metodología del Aprendizaje Basado en Problemas, entre los cuales se citan:

En España en la Universidad de Valladolid se desarrolló una investigación cuyo objetivo fue evaluar la adquisición de competencias del estudiantado de enfermería aplicando el Aprendizaje Basado en Problemas González, Villamor, Carbonero y Lara, (2013). Se emplearon los métodos descriptivo y transversal, además, se aplicaron técnicas como cuestionarios de evaluación a pares, autoevaluación.

Otra investigación realizada en México tuvo como propósito El Aprendizaje Basado en Problemas (ABP) una de las metodologías educativas que han tenido buena aceptación en instituciones universitarias en México. Donde se analiza la esencia del ABP, que consiste en identificar, describir, analizar y resolver problemas, lo cual se logra con la ayuda del docente, la metodología utilizada fue la investigación acción, que implicó un trabajo de campo caracterizado por la observación y participación de estudiantes universitarios, los instrumentos utilizados para la recolección de datos como las notas de campo, notas de los equipos, fotografías, un post-test, autoevaluación de los estudiantes, evaluación docente y de material didáctico según lo manifiestan Castaño, Víctor; Montante, Mario. (2015)

En la Unidad Educativa Pujilí, Ecuador se desarrolló una investigación cuyo objetivo fue precisar los conceptos teóricos, pedagógicos y didácticos que permiten la interpretación de la metodología del ABP enfocado desde diversas teorías, así como las definiciones existentes y sus aspectos fundamentales, realizando consultas bibliográficas en revistas como Redalyc, Scielo, Latindex entre otras, de igual manera en repositorios de universidades nacionales e internacionales empleando el Aprendizaje basado en problemas aplicado en Matemática Cadena (2020).

En la Universidad Estatal del Sur de Manabí también se desarrolló una investigación sobre el ABP, con el objetivo de que el estudiante aprenda a resolver problemas de ingeniería mediante la búsqueda sistemática de información y el razonamiento científico, desarrollando problemas vinculados a las ciencias agropecuarias aplicando los contenidos estudiados en el programa de estudio del segundo semestre de la carrera de Ingeniería Agropecuaria, utilizando para esto el método del Aprendizaje Basado en Problemas (ABP) y que el docente se convierta en un transmisor de conocimientos nuevos, como lo son los resultados de investigaciones recientes, para motivar al estudiante con temas de 
ingeniería actuales pero sin dejar de lado la teoría desde la que fueron desarrollados según Vera; Merchán; Zúñiga; Castro (2021)

El ABP es un proceso activo de aprendizaje que funciona a través de la solución de problemas relacionados con la interacción del hombre y su medio ambiente. La esencia del ABP consiste en identificar, describir, analizar y resolver tales problemas, lo cual se logra mediante la interacción del docente y los estudiantes.

Una vez establecida esta práctica, el siguiente paso es implementar los fundamentos pedagógicos en la formación de los estudiantes: aprender a ser, aprender a hacer, aprender a conocer y aprender a vivir en colectivo. De este modo se logrará que los estudiantes adquieran las habilidades necesarias para las competencias básicas de su profesión. Alfonso Roca, M.T. (2003)

1. Se pone especial énfasis en la relación docente-estudiante, la cual se busca sea intensamente interpersonal para que los estudiantes aprendan del docente.

2. En la práctica, el estudiante debe aprender los temas de sus cursos mediante proyectos de investigación fundamentados en disciplinas del conocimiento relacionadas con su profesión.

3. Se busca que los estudiantes autoevalúen su aprendizaje, sustentándose en una postura autodidacta, la búsqueda efectiva de información y el trabajo cooperativo dentro de un equipo, entre otros tantos aspectos que ayudan a satisfacer las demandas de la sociedad. Maudsley, G.; Strivens, J. (2000)

El objetivo de esta investigación está dirigido a que el estudiante aprenda a resolver problemas mediante la búsqueda sistemática de información y el razonamiento científico, desarrollando temas contenidos en los programas de los cursos de matemáticas, utilizando para esto la Metodología del Aprendizaje Basado en Problemas (ABP).

\section{Materiales y Métodos.}

Está investigación se llevó a cabo por medio de un estudio longitudinal descriptivo, prospectivo y cuantitativo basado en los métodos teóricos (análisis y síntesis), empíricos (técnica observación y encuestas) y métodos estadísticos para determinar la muestra y tabular los resultados del instrumento 
aplicado, la muestra está compuesta por 20 docentes de diferentes especialidades y enseñanzas, con el fin de evaluar la aplicación de la Metodología del Aprendizaje Basado en Problemas en el desarrollo del proceso de enseñanza-aprendizaje, utilizando investigaciones y bibliografías sobre el tema objeto de estudio.

\section{Resultados}

El cuestionario fue enviado a 20 docentes. De ellos 9 afirmaron haber aplicado la metodología el Aprendizaje Basado en problemas en sus clases, por tanto, constituirían nuestra muestra para el estudio. Las tres primeras preguntas tienen carácter informativo y reflejan los siguientes resultados.

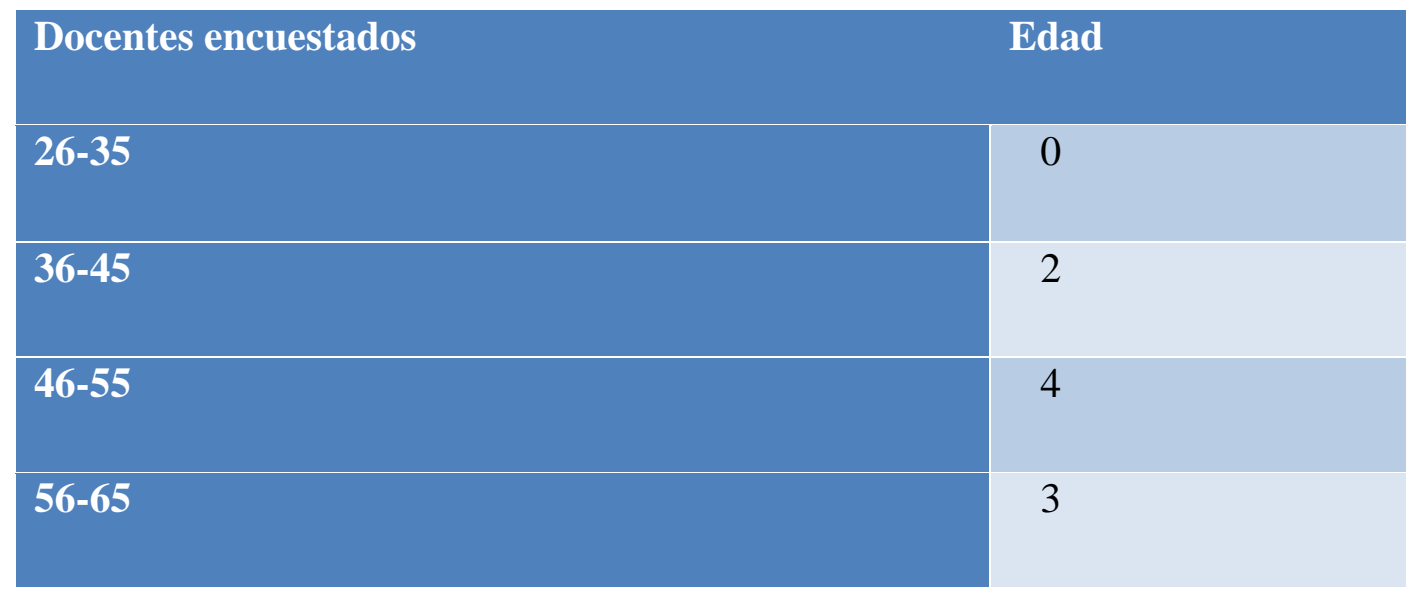

Tabla \# 1. Edad de los encuestados

\begin{tabular}{|lc|}
\hline Nivel en que trabajan los docentes encuestados & \\
\hline Bachillerato & $\mathbf{1}$ \\
\hline Nivelación & $\mathbf{1}$ \\
\hline Universitario & $\mathbf{6}$ \\
\hline Formación profesional & $\mathbf{1}$ \\
\hline
\end{tabular}

Tabla \# 2. Nivel en el que ejercen la docencia los encuestados 


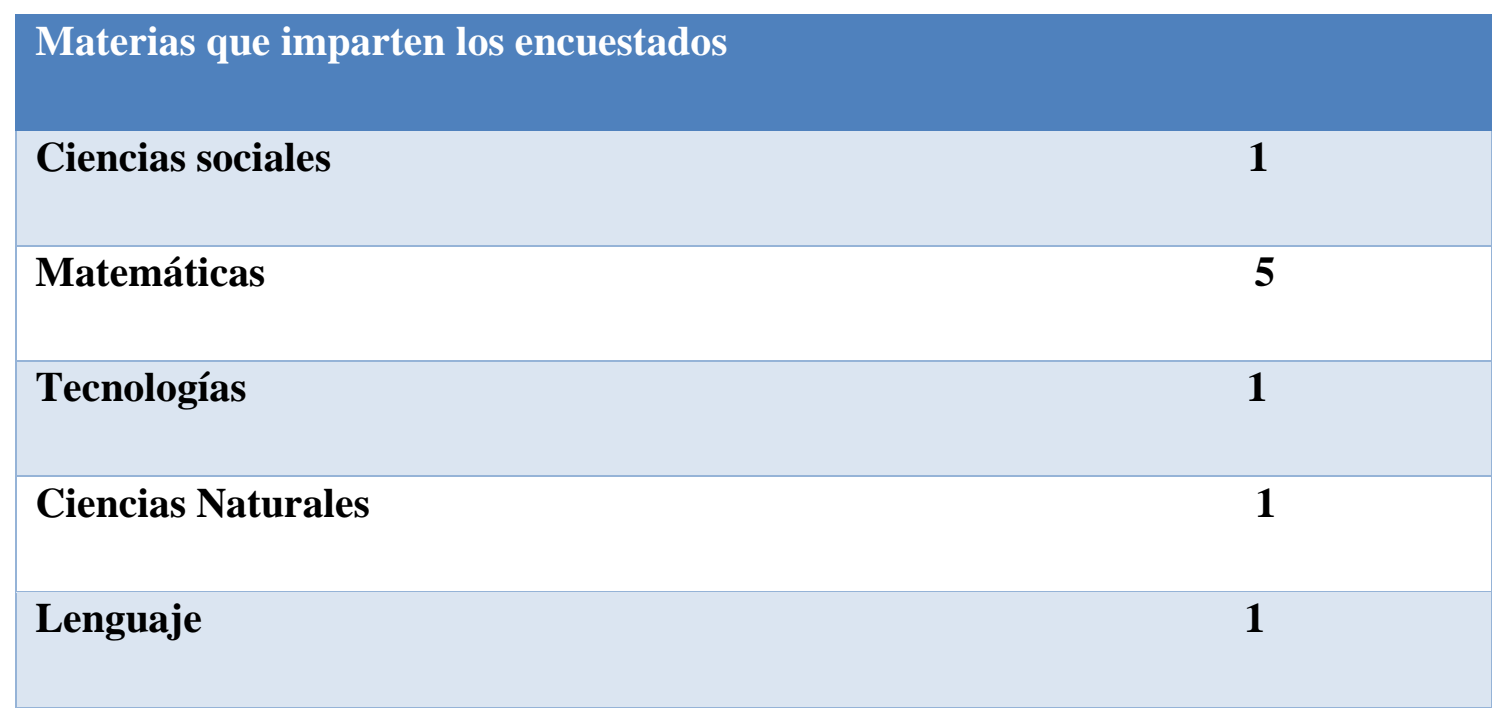

Tabla \# 3. Materias que imparten los encuestados

En la valoración global del uso del Aprendizaje Basado en Problemas podemos resaltar que los resultados fueron excelentes, tanto en las impresiones sobre la experiencia (gráfico \# 1) como su uso en las diferentes enseñanzas.

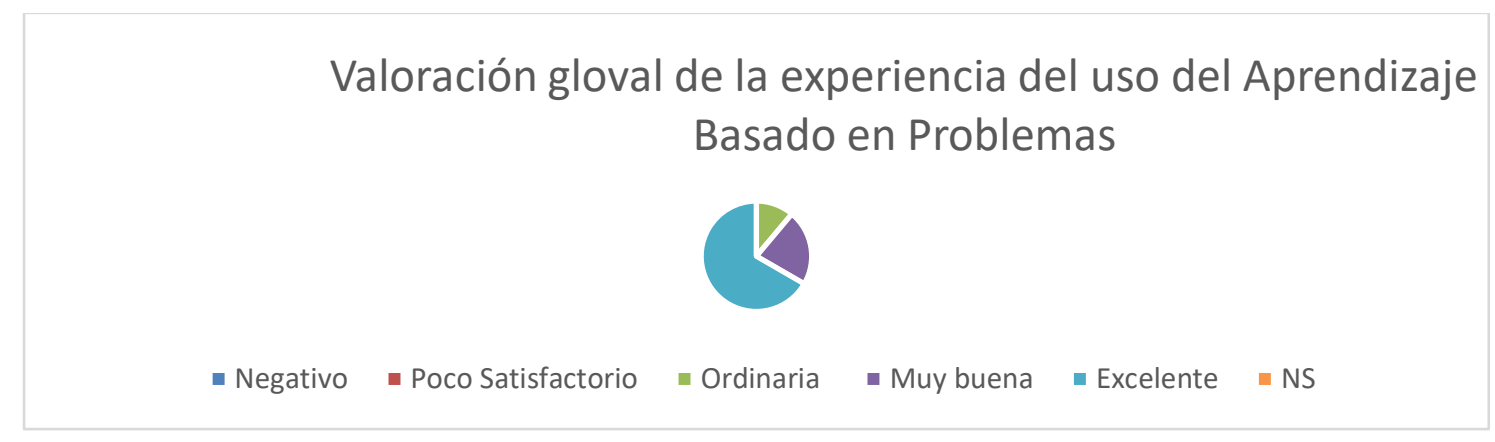

Gráfico \# 1. Valoración global sobre la experiencia del uso del Aprendizaje Basado en Problemas.

Analizando los aspectos principales del Aprendizaje Basado en Problemas (Tabla \# 4), los resultados son muy buenos, del total de las respuestas un promedio de respuestas 5 fueron muy buenas, en los diferentes indicadores, promedio de respuestas 3 adecuadas y promedio de respuestas, 1 poco satisfactorio y ninguno lo considero contrario o negativo. Desglosando los diferentes indicadores vemos que los resultados son buenos, en cuanto a la consecución de los objetivos, la motivación y la adecuación de la evaluación. De sobresaliente se puede calificar la colaboración de los estudiantes, la significación de los contenidos y la adquisición de las competencias básicas. 


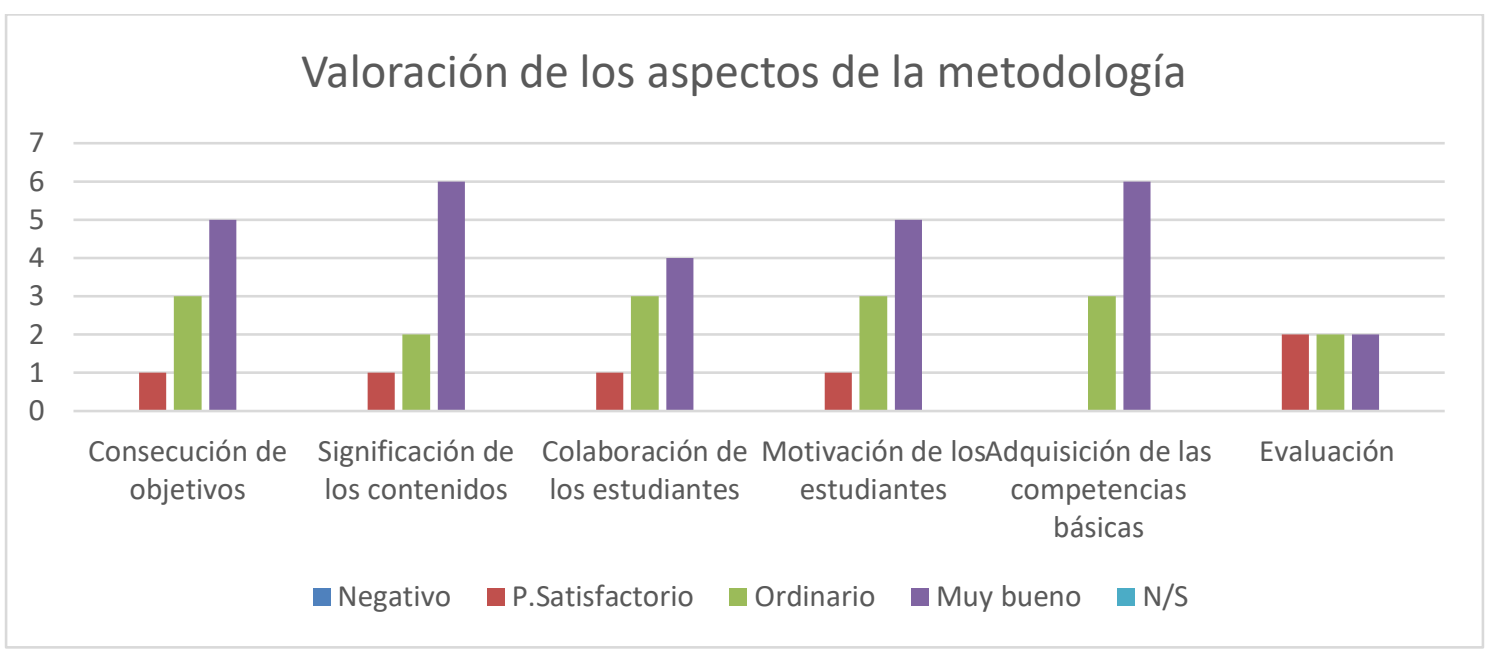

Tabla \# 4. Pregunta integradora para recoger la valoración sobre diferentes aspectos de la metodología.

En cuanto a la colaboración entre profesores se observa que los resultados son muy buenos, 4 lo consideran muy bueno, 3 adecuado y solo 2 poco satisfactorio, las relaciones entre las diferentes disciplinas para contribuir a lograr con una buena motivación de los estudiantes es un trabajo significativo, colaborativo y con mayor adquisición de competencias en los estudiantes, estimulándolos a la búsqueda, aprender a aprender y a la metacognición. Tabla \# 5

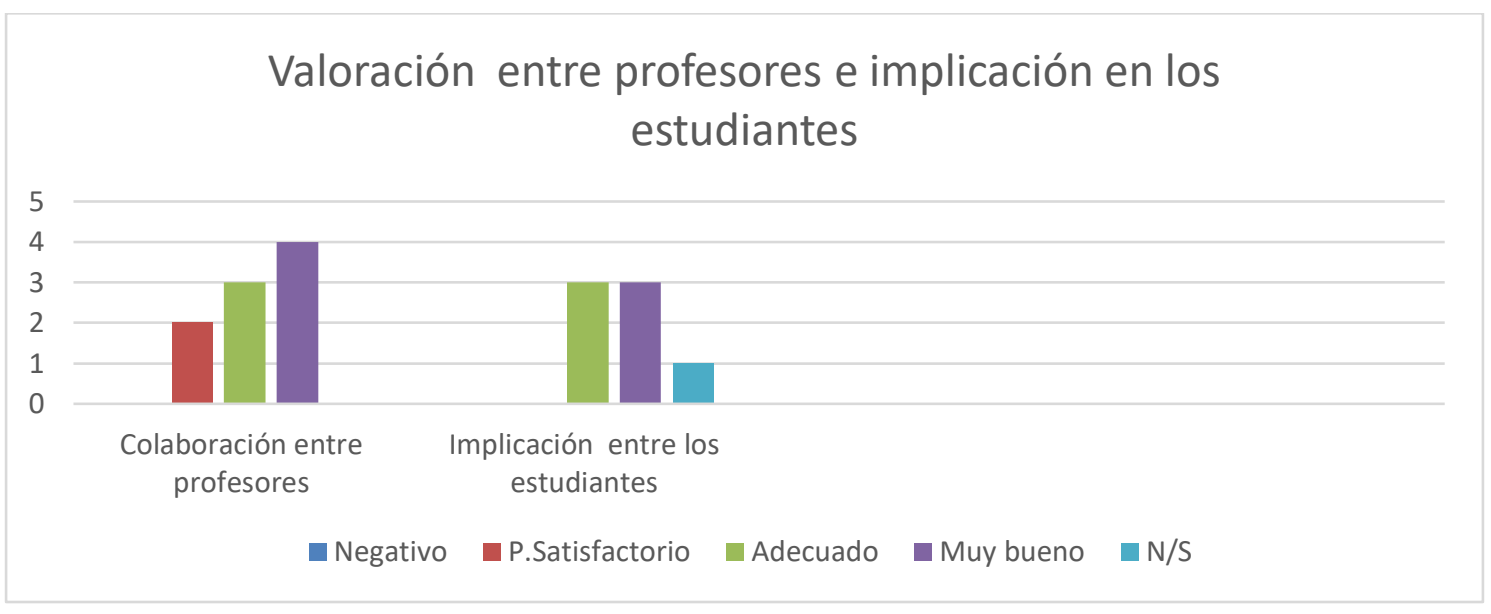

Tabla \# 5. Valoración entre los profesores e implicación en los estudiantes.

En el indicador sobre la valoración y uso del Aprendizaje Basado en Problemas en la mejora del proceso de enseñanza-aprendizaje se pudo observar que, 8 lo consideran muy importante, 2 importante 
y ninguno lo considera poco importante lo que demuestra que se considera muy importante para la mejora del proceso de enseñanza-aprendizaje coincidiendo con el estudio bibliográfico sobre las ventajas que tiene la utilización del Aprendizaje Basado en Problemas para la mejora del proceso de enseñanza-aprendizaje estimulando el trabajo colaborativo, la búsqueda, aprender-aprender y la metacognición. Gráfico \# 2.

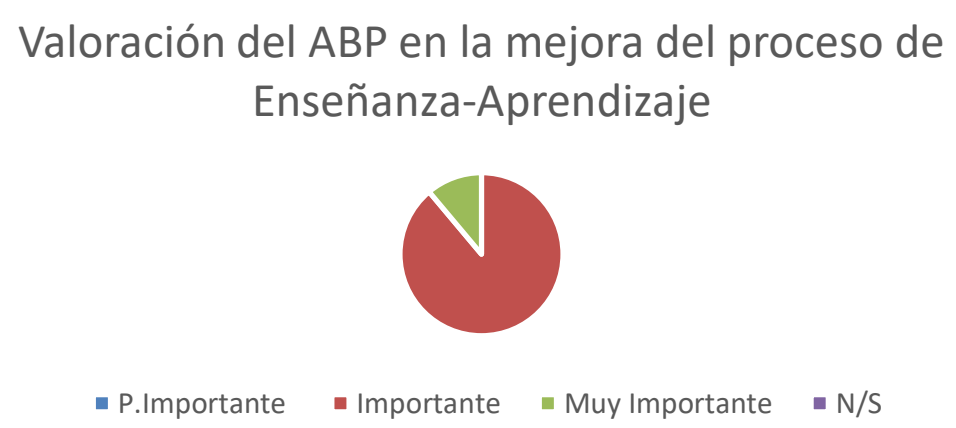

Gráfico \# 2. Valoración del uso del Aprendizaje Basado en Problemas en la mejora del proceso de enseñanza-Aprendizaje.

\section{Discusión}

Coincidimos con (Curay, 2013) en las ventajas del ABP relacionadas a continuación:

- $\quad$ Estudiantes con mayor motivación: El método estimula a los estudiantes a involucrarse más en el aprendizaje debido a que sienten que tienen la posibilidad de interactuar con la realidad y observar los resultados de dicha interacción.

- El aprendizaje es más significativo: Los estudiantes comprenden para qué se requiere aprender, cómo se relaciona lo que se hace y aprende con la realidad de su contexto.

- Desarrollo de pensamiento crítico y creativo: La misma dinámica del proceso en el ABP permite que los estudiantes adquieran habilidades y destrezas.

- La retención de información es mayor al aplicar problemas relacionados con la realidad de los estudiantes, el aprendizaje es más significativa para ellos. 
- Integración del conocimiento: Permite interrelacionar distintas disciplinas para dar solución al problema que se presente de manera integral.

- Mejora la comprensión y adquisición de habilidades: mediante la aplicación de problemas de su contexto y aumentan los niveles de comprensión.

- Habilidades interpersonales y de trabajo en equipo: El ABP promueve la interacción incrementando algunas habilidades como; trabajo colaborativo, evaluación de compañeros y cómo presentar y defender sus trabajos.

- Motivación: a través de los problemas que se aplican, relacionados con su vida diaria el estudiante se siente incentivado y presta mejor atención.

Es cierto lo que indica Planas (2011), que en ocasiones las metodologías como el ABP son difíciles de implementar y que debe hacerse en combinación con otras estrategias. Además, puede requerirse tiempo para que los estudiantes optimicen sus aprendizajes.

Sin embargo, el papel del profesor se presenta como un factor de máxima transcendencia, la función del docente es fundamental, en este sentido para Pehkonen (2007) la función del docente es fundamental y cuestiona que el poco uso del ABP a las exigencias que implica a los docentes y también señala como un factor clave y de grandes influencias sobre las actitudes y comprensión de los estudiantes.

De igual forma Savery (2006) considera que le cambio del roll del profesor representa una de las mayores dificultades a la hora de implementar el ABP y también señalan como factor clave en el desarrollo de la metodología y de gran influencia sobre las actitudes y comprensión de los estudiantes.

Como resultados de la investigación bibliográfica y la encuesta aplicada se pudieron apreciar que trabajar con la metodología del ABP provoca que las actividades realizadas por los estudiantes estén relacionadas con problemas de la disciplina y de la vida real. Que sean desarrolladas en un ambiente colaborativo donde cada estudiante dentro de su equipo desempeñe una tarea específica y siguiendo la metodología dividida en etapas cuyas competencias de la disciplina en matemáticas eran 
constantemente utilizadas por los estudiantes desde proponer sus diferentes formas de solucionar el problema, seleccionar una idea y a partir de ella construir su modelo matemático.

\section{Conclusiones.}

1-Las matemáticas, debido a su carácter abstracto, necesitan de ambientes propios, que estimulen en el estudiante el desarrollo del pensamiento matemático y faciliten el proceso de enseñanzaaprendizaje, además, requieren de estrategias didáctico-pedagógicas y metodologías que despierten la curiosidad, el interés y el gusto por la materia.

2. La aplicación del ABP en el aprendizaje de la matemática es de gran utilidad, puesto que permite a los estudiantes realizar diversas propuestas para solucionar un problema, mediante la motivación, aprendizaje significativo, desarrollo de habilidades de pensamiento crítico y creativo, permitiendo mejorar la comprensión, estimulándolos a la búsqueda, aprender a aprender y a la metacognición.

3. Luego de analizar los diferentes contextos tanto a nivel nacional como internacional se puede deducir que el ABP es una metodología sencilla centrada en el aprendizaje que permite al estudiante resolver de forma autónoma problemas, mediante el desarrollo de destrezas, habilidades y actitudes necesarias para construir y aplicar de forma eficaz el conocimiento y vincularlo con su desarrollo profesional para la mejora del proceso de enseñanza-aprendizaje estimulando el trabajo colaborativo y la búsqueda.

\section{Bibliografía.}

Alfonso Roca, M.T. (2003) Metodología Docente e Innovación Pedagógica. En: I Congreso Nacional de Calidad de la Enseñanza en Fisioterapia. Facilitando el aprendizaje: 810 de mayo de 2003. Murcia: Universidad de Murcia.

Ausubel, D. (1986) .Educational Psychology. A Cognitive View, Holt, Rinehert and Winston, N.York, Trillas, México. 
Bonilla, M. (2015). Propuesta metodológica para el aprendizaje significativo de química experimental en las y los estudiantes que acuden a la unidad de química de la universidad central del ecuador (UCE). http://repositorio.puce.edu.ec/handle/22000/10577

Cadena Zambrano, Verónica E. (2020). Aprendizaje basado en problemas aplicado en Matemática. Revista Científico Educacional La Roca RPNS: 2090 | ISSN: 2074-0735 ROCA Universidad de Granma, Cuba.

Castaño, Víctor; Montante, Mario. (2015). El método del Aprendizaje Basado en Problemas como una Herramienta para la Enseñanza de las Matemáticas. Revista Iberoamericana para la Investigación y el Desarrollo Educativo, vol. 6, núm. 11, julio-diciembre, 2015. Centro de Estudios e Investigaciones para el Desarrollo Docente A.C. Guadalajara, México. Disponible en https://www.redalyc.org/articulo.oa?id=498150319022

Curay, E. (2013). Incidencia de la estrategia del aprendizaje basado en problemas en el rendimiento académico en Matemática de los estudiantes de noveno año de Educación Básica del Colegio Nacional Primero de Abril del cantón de Latacunga (Trabajo de investigación Previa a la obtención del Grado Académico de Magíster en Docencia Matemática, Universidad Técnica de Ambato, Ambato, Ecuador). http://repositorio.uta.edu.ec/jspui/handle/123456789/5768

González, H., Villamor, M. \& Carbonero M. \& Lara F. (2013). Evaluación por competencias de los estudiantes de Enfermería a través de Aprendizaje Basado en Problemas. Enfermería Universitaria, 10 (4), 120-124. Recuperado el 1 de octubre del 2018 de http://www.redalyc.org/articulo.oa?id=358733529003

Mancheno, T. (2013). Aplicación del aprendizaje basado en problemas como estrategia didáctica en la educación superior. (Tesis previo a la obtención del título de magíster en docencia universitaria en las ciencias administrativas y económicas, UNIANDES, Ambato, Ecuador). http://dspace.uniandes.edu.ec/handle/123456789/4536

Martínez, H. (2014). La aplicación del aprendizaje basado en problemas (ABP) como estrategia para potenciar el aprendizaje académico en el módulo de algebra con los estudiantes de primer semestre de la facultad de ingeniería en sistemas electrónica e industrial de la Universidad Técnica de Ambato (Trabajo de Titulación Previo a la obtención del Grado Académico de Magíster en Docencia 
Matemática).Recuperado

el

29

de

septiembre

del

2018

dehttp://repositorio.uta.edu.ec/jspui/handle/123456789/7459

Matamoros, W. (2018) Propuesta didáctica de aprendizaje basado en problemas dirigida al área de matemáticas ( $8^{\circ}$ de educación general básica): caso Unidad Educativa "Sagrada Familia". (Tesis Maestría en Ciencias de la Educación, Pontificia Universidad Católicadel Ecuador, Quito, Ecuador). Recuperado el 29 de septiembre del 2018 de http://repositorio.puce.edu.ec/handle/22000/15115

Maudsley, G.; Strivens, J. (2000) Promoting professional knowledge, experiential learning and critical thinking for medical students. Medical Education. 34, (7) pp. 535-544.

Pehkonen, E (2007). Problem Solving in Mathematics Educations in Finland.Université de Genéve Disponible en: http://www.unige.ch/math/EnsMath/Rome2008/WG2/Papers/PEHKON.ppt

Rojas, J., Urdaneta, E., \& Guevara, L. (2014). Estrategias para el aprendizaje significativo de procesos de fabricación mediante orientación constructivista. Opción: Revista de Ciencias Humanas y Sociales, (75), 92-103. https://dialnet.unirioja.es/descarga/articulo/5140072.pdf

Savery, J. R. (2006). Overview of Problem-based Learning: Definitions and Distinctions.Interdisciplinary Journal of Problem-Based Learning, 1. Available at: https://doi.org/10.7771/1541-5015.1002

Vera Velázquez, Raquel; Merchán García, William; Maldonado Suñiga, Kirenia; Castro Landin, Alfredo. (2021). Metodología del aprendizaje basado en problemas aplicada en la enseñanza de las Matemáticas. Serie Científica de la Universidad de las Ciencias Informáticas. La Habana, Cuba. Vol. 14, No. 3 Mes: Marzo, 2021, Pág. 142-155. http://publicaciones.uci.cu.

Vigotsky, Liev Semionovich (1960). El desarrollo de las funciones psíquicas superiores. Moscú: Academia de Ciencias Pedagógicas de RSFSR. http://www.taringa.net/perfil/vygotsky 